\title{
The Impact of Abductor and Adductor Contraction in a Bridging Exercise on Muscle Activities in of the Abdominal Region and the Lower Extremities
}

\author{
SAng-Yeol Lee, PT, PhD ${ }^{1)}$, Su-Kyoung Lee, PT, MS ${ }^{2}$ \\ 1) Department of Physical Therapy, Kyungsung University \\ 2) Department of Physical Therapy, Gimhae College: Sambang-Dong, Gimhae-si, Gyungnam 621-706, \\ Republic of Korea. TEL: +82 55-3201735, FAX: +82 55-3366222,E-mail: holeintwo@naver.com
}

\begin{abstract}
Purpose] This study examined the effects of different bridging exercises on the trunk muscles, and provides preliminary data for clinical use. [Methods] Bridging exercise with hip abduction and adduction were used to examine the muscle activation of the hip abductors, which were measured by EMG. The subjects were 45 healthy young males. [Results] During adduction there were from general bridging position in all the muscle activities except for RF. The highest muscle activities were seen in TrA, EO and AM during adduction, and in RA and GM during abduction. [Conclusion] Bridging exercise with isometric abduction of the hip joint is effective at contracting RA and GM. Stabilizer exercise, it seems possible selectively strengthening of specific particular muscles by isometric adduction or abduction of the hip joint appears possible in bridging exercises for people who need lumbar stabilization exercises
\end{abstract}

Key words: Bridging exercise, Muscle activation, Trunk stabilization exercise

(This article was submitted May 10, 2012, and was accepted Jun. 13, 2012)

\section{INTRODUCTION}

Overall coordination of the trunk muscles is more important than activation of particular muscles for the stabilization of spine ${ }^{1)}$. Recently, studies have been conducted on posture and movement control, focusing on core stability 2,3 ). Core stability exercise helps to control force when a patient's posture is instable, and enables conscious and automatical control of movement, allowing maintenance of the spine neutral position, the best position for dealing with external loading. This exercise is receiving growing attention as a preventive measure along with therapeutic exercise ${ }^{4)}$. Among various stabilization exercises, the bridging exercise has an important finfluence on functions of pelvic movement, which are involved in mobilization on the bed, use of a toilet seat, relief of pressure, putting on clothes on the lower limbs and gait $^{5)}$. Also, pelvic movement is important for weightbearing on the feet and in the half kneeling position. In a sitting position, the core stability exercise increases postural control in the standing position, and strengthens the lower spine and hip extensor muscles in preparation for the stance phase of a gait ${ }^{5}$.

Stabilization of the trunk enhances functional posture and increases spine and pelvic stability in movement. It also strengthens muscles and helps to improve control of the muscles and balance in movements ${ }^{6}$. Among the methods that gradually increase intensity of trunk stabilization, one method increases the intensity and frequency of resistance, and another method increases exercise intensity while raising instability of the support surface using a therapeutic ball, vestibular balance board or a form roll ${ }^{7}$.

Studies of muscle loading and spinal stability have reported that, when a healthy adult performs a lumbar stabilization exercise, there is high correlation with abdominal curl, side bridging exercise with right isometric side support, bridging exercise with right leg raise, bridging exercise, and the muscle activity of the rectus abdominalis ${ }^{8}$. Also, it was reported that exercise of the abdominal muscle to maintain the neutral positions for maintenance of the spine in the during external loading with trunk rotation minimizes contraction of the rectus abdominis and causes co-contraction of the transverse abdominis and the oblique muscle, and is thus suitable as a trunk stabilization exercise ${ }^{9)}$.

Activation of the abdominal muscles is critical for stabilizing the pelvic muscles against the pulling force of the hip muscles. When the pelvic muscle is stable, the forces on the trunk are transmitted efficiently to the hip joint and the lower extremities ${ }^{10}$ ). In this study, we examined how the hip joint and the lower extremities that are stabilized in an isometric adduction, and abduction, affect the activity of the abdominal muscles in a bridging exercise.

\section{SUBJECTS AND METHODS}

The participants were forty-five healthy males in their $20 \mathrm{~s}$ and $30 \mathrm{~s}$, who had sufficient muscle strength, range of motion and balance ability to perform the bridging exercise, and were mentally capable of understanding and agreeing to participation in the experiment. Those who had problems in the nervous system, cardiopulmonary system or musculo- 
Table 1. Comparison of muscle activities among the different bridging exercise positions

\begin{tabular}{lllc}
\hline Muscle & \multicolumn{1}{c}{ Neutral } & Adduction & Abduction \\
\hline TrA* & $355.40 \pm 56.63$ & $766.31 \pm 145.79^{\dagger}$ & $513.51 \pm 79.77$ \\
RA* $^{*}$ & $166.26 \pm 5.56$ & $188.67 \pm 10.00$ & $204.35 \pm 8.53^{\ddagger}$ \\
OO $^{*}$ & $228.84 \pm 15.77$ & $344.20 \pm 38.91^{\dagger}$ & $320.49 \pm 27.46^{\ddagger}$ \\
AM$^{*}$ & $171.94 \pm 12.27$ & $433.82 \pm 41.78^{\dagger}$ & $226.82 \pm 27.51^{\ddagger}$ \\
$\mathrm{GM}^{*}$ & $643.55 \pm 111.67$ & $829.03 \pm 184.12$ & $1205.85 \pm 166.96^{\ddagger}$ \\
RF & $329.30 \pm 95.15$ & $460.81 \pm 118.86$ & $473.23 \pm 148.67$ \\
\hline
\end{tabular}

unit=\%RVC. mean \pm SE. TrA: Transversus Abdominis. RA: Rectus Abdominis. EO: External Obliquus. AM: Adductor Magnus . GM: Gluteus Medius . RF: Rectus Femoris. Superscripts: †significant difference between neutral and adduction, `significant difference between neutral and abduction, ${ }^{\ddagger}$ significant difference between adduction and abduction

skeletal system of the trunk and the lower extremities were excluded.

When performing the bridging exercise, the participants took the same starting position as the core stabilization exercise. They spread the knees to shoulder width, aligning the feet on a line extended from the shoulder to the knee. They were asked to maintain the distance between the knee and the feet during the exercise. The head and the eyes faced the ceiling so as not to affect the posture during the exercise. The general bridging exercise was performed for the first method, and a bridging exercise with isometric hip adduction was performed for the second method, keeping an exercise ball between the knees to adduct the hip joint.

For the third method, the participants performed a bridging exercise with isometric hip joint abduction, keeping an exercise ball between the knees and a wall to abduct the hip joint. Each exercise was repeated three times, in a random order. Each posture was kept for five seconds, and the muscle activation data for the middle three seconds, excluding the first and the last seconds, were recorded and averaged for the analysis. To prevent fatigue, the participants took one-minute break after each five-second exercise.

To examine how contraction of the hip joint in isometric adduction and abduction during the bridging exercise affected the muscle activities in the abdominal region and the lower extremities, a surface EMG Biopac student Lab MP 36 (Biopac System Inc, USA) was used. Data were filtered and processed on a computer using Acknowledge software. To reduce measurement errors, skin at the electrode placement site was cleansed with alcohol, then the electrodes were attached using a sufficient amount of electrolyte. From the measured EMG signals of the muscles, RMS values were calculated. These were divided by the resting voluntary contraction (RVC) value and are presented as percentagevalue $(\% \mathrm{RVC})$.

To measure the subjects' muscle activities of the transverse abdominis (TrA), rectus abdominis (RA), external oblique (EO), adductor magnus (AM), gluteus medius (GM), and rectus femoris (RF), surface electrodes were attached to the trunk and the low extremity of the dominant side ${ }^{11}$.

The experimentel results were analyzed using SPSS Version 18.0 for Windows. To examine differences in muscle activities between the general bridging exercise and the bridging exercise isometric hip adduction and abduction, repeated one-way ANOVA was used. Turkey's test was used for a post hoc test, and the significance level, $\alpha$, was chosen as 0.05 .

\section{RESULTS}

The general bridging exercise and bridging exercises with isometric hip adduction and abduction were performed to examine differences in muscle activities. During adduction and abduction there were significant differences $(\alpha<0.05)$ from the general bridging position in all the muscle activities except for RF. The highest muscle activities were seen in TrA, EO and AM during adduction, and in RA and GM during abduction (Table 1).

\section{DISCUSSION}

Activity of the abdominal muscles is necessary to stabilize the pelvis against the pulling forces of hip muscles. In a stabilized state, the forces of the trunk on the trunk are efficiently transmitted to the hip joint and the lower extremities ${ }^{10)}$. The stability of the trunk increases stability of the vertebrae and the pelvis in functional postures and movements, strengthens muscle power, and improve control and balance of the muscles and movements ${ }^{6}$. Among various stabilization exercises, the bridging exercise has an important influence on functions of pelvic movement which are used in mobilization on the bed, use of a toilet seat, relief of pressure, putting on clothes on the lower limbs and gait ${ }^{5}$. This study examined how the isometric adduction and abduction of the hip joint in a bridging exercise affected muscle activities the abdominal region and the lower extremity.

During adduction and abduction were significant differences $(\alpha<0.05)$ from the general bridging position in all the muscle activities except for RF. The highest muscle activities were seen in TrA, EO and AM during adduction, and in RA and GM during abduction. The contraction of the AM muscle along with active of muscle activities of TA and EO seems to indicate that the TrA and EO muscles co-contract for stable performance of the bridging exercise. This would increase the abdominal pressure, and compress the organs in the abdominal region to increase lumbar and trunk stability, and it would also increase abdominal pressure, resisting hyperlordosis. In addition, the transverse abdominis is the 
most active muscle in isometric trunk extension, and it is the most consistent muscle under changing abdominal pressure to increase the vertebral stability ${ }^{12)}$.

The core principle of the bridging exercise is that it controls external force on the lumbar region, and the coordination and interaction of the muscles around the arms and legs in their movements in order to prevent disc injury, or repeated impairment of the vertebral facet or other tissues in the area. More specifically, the bridging exercise increases abdominal pressure, by efficiently performing lumbar stabilization training, and induces muscle co-contraction to reduce hyperlordsis or anterior pelvic tilt ${ }^{13)}$. In comparison, a bridging exercise with contraction and abduction of GM contributes to pelvic stability, resisting widening of the pubic due to abduction of bilateral RA. In a pelvic tilting motion, the lower rectus abdominis and lower part of the gluteus are activated, while the activity of the multifidus is reduced $^{14)}$. Therefore, spinal stabilization exercises should include recruitment of the lower half of the rectus abdominis and lower parts of the gluteus in its actions, because correcting pelvic tilt is an important skill which is required for improving stability. The RF muscles were most active in abduction but the increase in activity was not significant. $\mathrm{RF}$ is a two joint muscle controlling hip flexion and knee extension, and since the bridging exercise involves opposite motions, it did not significantly affect the RF muscles.

\section{ACKNOWLEDGEMENT}

This research was supported by Kyung-sung University
Research Grants in 2012.

\section{REFERENCES}

1) Breen AC, Muggleton JM, Mellor FE, et al.: Trunk muscle activity in healthy subjects during bridging stabilization exercises. BMC Musculoskelet Disord, 2006, 7: 1-8. [Medline] [CrossRef]

2) Akuthota V, Nadler SF: Core strengthening. Arch Phys Med Rehabil, 2004, 85: S86-S92. [Medline] [CrossRef]

3) Page P: Sensorimotor training: A "global" approach for balance training. J Bodyw Mov Ther, 2006, 10: 77-84. [CrossRef]

4) Magee DJ: Instability and Stabilization: Theory and treatment (2nd ed). Seminar Workbook, 1999.

5) O'Sullivan SB, Schmitz TJ: Physical Rehabilitation: Assessment and Treatment (4th ed). Philadelphia: F.A. Davis Company, 2001, pp 245-255.

6) Richardson CA, Snijders CJ, Hides JA, et al.: The relation between the transverses abdominis muscle, sacroiliac joint mechanics, and low back pain. Spine, 2002, 27: 399-405. [Medline] [CrossRef]

7) Hall CM, Brody LT: Therapeutic Exercise: Moving Toward Function (1st ed). Philadelphia: Lippincott Williams \& Wilkins, 1999, pp 130-141.

8) Kavcic N, Grenier S, McGill SM: Quantifying tissue loads and spine stability while performing commonly prescribed low back stabilization exercises. Spine, 2004, 29: 2319-2329. [Medline] [CrossRef]

9) Richardson C, Toppenberg T, Jull G: An initial evaluation of eight abdominal exercise for their ability to provide stabilisatio for the lumbar spine. Aust J Physiother, 1990, 36: 6-11.

10) Neumann DA: Kinesiology of the Musculoskeletal System: Foundations for Physical Rehabilitation (1st ed). St Mosby, 2002, pp 214-231.

11) Cram JR, Kasman GS, Holtz J : Introduction to Surface Electromyography. Maryland: Aspen Pub, 1998, pp 121-130.

12) Norris CM: An exercise programme to enhance lumbar stabilization. Physiotherapy, 1995, 81: 138-145. [CrossRef]

13) Kisner C, Colby LA: Therapeutic Exercise Foundations and Techniques (5th ed). Philadelphia: F. A. Davis, 2007, pp 300-320.

14) Richardson C, Jull G, Toppenberg R, et al.: Techniques for active lumbar stabilisation for spinal protection: a pilot study. Aust J Physiother, 1992, 38: $105-112$. 